\title{
ERRATUM
}

\section{Gabor filters and phase portraits for the detection of architectural distortion in mammograms}

\author{
Rangaraj M. Rangayyan · Fábio J. Ayres
}

Published online: 23 September 2006

(C) International Federation for Medical and Biological Engineering 2006

\section{Erratum to: Med Biol Eng Comput DOI 10.1007/s11517-006-0088-3}

Due to a processing error the presentation of the sixth sequence of Sect. 2.1 and Eqs. 8 and 10 was incorrect. The correct versions are given below.

Let $\theta(x, y)$ be the texture orientation at $(x, y)$, and $g_{k}(x, y), k=0,1, \ldots, 179$, be the Gabor filter oriented at $\alpha_{k}=-\pi / 2+\pi k / 180$. Let $I_{\mathrm{HPF}}(x, y)$ be the high-pass-filtered version of the mammogram being processed, and $I_{k}(x, y)=\left(I_{\mathrm{HPF}} * g_{k}\right)(x, y)$ represent the Gabor-filtered images, where the asterisk denotes linear convolution. Then, the orientation field angle of $I(x, y)$ is given by

$\theta(x, y)=\alpha_{k_{\max }}$ where $k_{\max }=\arg \left\{\max _{k}\left[\left|I_{k}(x, y)\right|\right]\right\}$.
The online version of the original article can be found at http://dx.doi.org/10.1007/s11517-006-0088-3.

R. M. Rangayyan $(\bowtie)$. F. J. Ayres

Department of Electrical and Computer Engineering,

Schulich School of Engineering, University of Calgary,

Calgary, AB, Canada

e-mail: ranga@ucalgary.ca

R. M. Rangayyan

Department of Radiology, University of Calgary,

2500 University Drive N. W., Calgary, AB,

Canada T2N 1N4

$$
\begin{aligned}
& \theta_{\mathrm{f}}(x, y)=\frac{1}{2} \arctan \left(\frac{(h * s)(x, y)}{(h * c)(x, y)}\right), \\
& M_{\mathrm{f}}(x, y)=\left(h * M_{\mathrm{CLS}}\right)(x, y),
\end{aligned}
$$

